\title{
Characterisation of Cellulose Nanofibres Derived from Chemical and Mechanical Treatments
}

\author{
Martini Muhamad ${ }^{1, a}$, Peter Hornsby ${ }^{2}$, Eugene Carmichael ${ }^{3}$, Muhammad Zakaria ${ }^{4}$, Yew Been Seok ${ }^{1}$, \\ Saiful Mohamed ${ }^{1}$ and Shekhar Sharma ${ }^{3}$ \\ ${ }^{1}$ Faculty of Innovative Design and Technology, Universiti Sultan Zainal Abidin, Kuala Terengganu, Malaysia \\ ${ }^{2}$ Polymer Cluster, Queen's University Belfast, School of Mechanical and Aerospace Engineering, Ashby Building, \\ Belfast, BT9 5 AH UK \\ ${ }^{3}$ Applied Plant Science Division, Agri-Food and Bioscience Institute, Newforge Lane, Belfast, BT9 5PX, UK. \\ ${ }^{4}$ Faculty of Manufacturing Engineering Technology, TATI University College, 24000 Kemaman, Terengganu, \\ Malaysia.
}

\begin{abstract}
Optimised routes have been established to obtain high aspect ratio cellulose microfibrils derived from plant feedstocks, involving a combination of a chemical and mechanical treatment using a high pressure microfluidizer. By this means, nano-cellulose was produced using different pretreatment protocols. The nanofibres produced were characterised for its particle size distribution measurement (laser diffraction and zeta potential analysis) and fourier transform infrared for its structural analysis. Results obtained and subsequent trends observed in chemical, mechanical and chemo-mechanical treated nanofibres were compared and contrasted. The chemomechanical treatment (TEMPO-oxidation and high pressure microfluidisation) yielded higher aspect ratio nanofibrils than nanofibrils made by solely chemical or mechanical treatment.
\end{abstract}

\section{Introduction}

There is increasing interest in the use of natural fibres as reinforcing additives for polymers, for example in medical and packaging applications. Nano-variants of these materials can have mechanical properties higher than conventional glass fibres, and potentially of the same order of carbon fibres. Furthermore, since they are derived from renewable sources this makes them more environmentally attractive. These natural fibres have relatively low cost with low density giving high specific mechanical properties to the composite. This type of material has attracted great interest from industry and academia due to the remarkable improvements in properties achievable at very low addition levels compared to virgin polymer or conventional micro- and macro-composites. Although the precise reasons for this improvement have been the subject of intense research, the smaller size of the reinforcing phase undoubtedly increases the probability of structural perfection with less surface defects thereby contributing to a more efficient reinforcement compared to micron-sized alternatives reinforcements [1].

A major drawback of using natural fibres (macro-fibres) for composite applications is resulting in large variation in properties inherent to natural products. The properties could be impacted by climatic

\footnotetext{
${ }^{\mathrm{a}}$ Corresponding author : martinimuhamad@unisza.edu.my
} 
conditions, maturity, soil type and also disturbance during plant growth. Therefore, the idea of decreasing the size of the natural fibre particles from micro to nanoscale has been applied to overcome this problem. The major benefit of using cellulose nanofibres in reinforcing polymer matrices is its high surface area which leads to a very large matrix-filler interfacial area, changing the molecular mobility, relaxation behavior and ensuring thermal and mechanical properties.

The cellulose nanofibres used in this study were derived from banana fibres. For many years, the trunk of the banana tree has been considered as a waste-product and as a result, research was started to evaluate the potential of utilizing the trunk for industrial purposes, in particular after extraction of fibres from the banana trunk. Banana fibre is a ligno-cellulosic fibre, obtained from the pseudo-stem of banana plant (Musa sepientum), which is classed as a bast fibre with relatively good mechanical properties [2]. In preliminary work, the average tensile stress for single banana fibre was recorded approximately $670 \mathrm{MPa}$.

In this study, work is presented and focused on the optimisation of the processing and derivation techniques to achieve the optimum yield of stable nanofibre suspensions by applying various methodologies: chemical, mechanical and chemo-mechanical treatments. Particular emphasis is given to the structural characterisation of these fibres, in order to define their suitability as potential reinforcements for polymers.

\section{Experimental}

\subsection{Materials}

Raw banana fibre obtained from the superposed layers of banana trees pseudostem were produced from COPLACA (Grupo Regional de Cooperativas Plataneros Del Archipielago) and then were processed into a long fine fibres at University of Las Palmas, Canary Island. The long fibres then were cut into short fibres at Grupo Antolin (Grupo Antolin Ingenieria S. A.) and then dried under shade. Average fineness of banana fibre is $2386 \mathrm{Nm}$ (4.1 dtex).

\subsection{Preparation of cellulose nanofibres}

The pre-processed banana fibres were dry-milled and pre-treated with sodium hydroxide, $\mathrm{NaOH}(1$ mol) for four hours at $80^{\circ} \mathrm{C}$. An important aim of this pre-treatment was to increase the surface area of the lignocellulosic material to make polysaccharides more susceptible to hydrolysis [3]. The fibres were then washed and freeze dried before up taken for further three different treatment methods.

\subsubsection{Acid hydrolysis}

Banana fibres were hydrolysed at different conditions. Pre-treated fibres were added to acid solution which contains $60 \mathrm{wt} \%$ of water, $10 \mathrm{wt} \%$ of concentrated hydrochloric acid $(\mathrm{HCl})$ and $30 \mathrm{wt} \%$ of concentrated sulphuric acid $\left(\mathrm{H}_{2} \mathrm{SO}_{4}\right)$. The mixture was heated to $80{ }^{\circ} \mathrm{C}$ for 16 hours with continuous stirring and then neutralized using $\mathrm{NaOH}$. The mixture was dialysed against running water for two days.

\subsubsection{Mechanical Treatment}

The cellulose nanofibres were prepared from banana fibres using a homogenisation process. The pretreated fibres were first added to de-ionized water and turraxed for 20 minutes at $10000 \mathrm{rpm}$. This procedure was carried out by T25 Digital ULTRA-TURRAX ${ }^{\circledR}$ in an ice bath in order to break up the particle size of banana fibres before going through in the microfluidizer. The microfluidiser M-110P is 
a laboratory unit machine that provides continuous high shear rate processing under high process pressure up to 2868 bar (30 $000 \mathrm{psi}$ ), with a flow rate up to $120 \mathrm{ml} / \mathrm{min}$ and sample size of $50 \mathrm{ml}$.

\subsubsection{Chemo-mechanical treatment}

Cellulose nanofibres were prepared using a combination of TEMPO oxidation and microfluidizer. The pretreated fibres were added to an aqueous solution containing $0.000125 \mathrm{wt} \%$ of TEMPO $(2,2,6,6$ Tetramethyl1-piperidinyloxy, free radical) and $0.00125 \mathrm{wt} \%$ sodium bromide. The reaction was initiated by adding $0.01 \mathrm{wt} \%$ sodium hypochlorite. All the procedures were held at room temperature. The neutral mixture was then passed through into microfluidizer for 10 passes through 200 and $87 \mu \mathrm{m}$ chamber at 30000 psi.

\subsection{Characterisation of cellulose nanofibres}

\subsubsection{Dynamic light scattering (DLS)}

Nanofibre suspension's particle size distribution was measured by DLS method with a Zatasizer ZS series from Malvern Instruments, England. Nano ZS measures the scattering information close to $180^{\circ}$ where the detector position is located at $173^{\circ}$. This is known as backscatter detection where the incident beam does not have to travel through the entire sample.

\subsubsection{Zeta potential analysis}

Cellulose nanofibres were analyzed by using a Zetasizer ZS series from Malvern Instruments, England. If the particles in suspension have a large negative or positive zeta potential then they will tend to repel each other and no tendency to flocculate. The general dividing line between stable and unstable suspension is generally taken either $=30 \mathrm{mV}$ or $-30 \mathrm{mV}$.

\subsubsection{FTIR analysis}

FTIR spectroscopy is able to provide evidence of any new functional groups, also investigate the chemical structure changes when treatments (chemical and mechanical) are applied. FTIR analysis was performed onto raw materials and cellulose nanofibres in order to study their changes in chemical structure by using attenuated total reflection (ATR) analysis. The FTIR spectra of all specimens were performed using a Perkin Elmer spectrometer (Spectrum 1000) instrument with a universal ATR sampling accessory. 64 scans were collected between 650 and $4000 \mathrm{~cm}^{-1}$ with $2 \mathrm{~cm}^{-1}$ resolution.

\section{Results and discussion}

\subsection{Size distribution of cellulose nanofibres}

Results from DLS determinations are shown in Figure 1, in terms of Z-average diameter. The cellulose nanofibres treated with acid hydrolysis shows a larger particle size compared to the other products, by means that the nanofibres particles treated with acid hydrolysis aggregated in aqueous suspension rapidly compared to other samples. These observations can be explained by higher hydrophobic characteristics of the particles i.e. lower number of $\mathrm{OH}$ groups in these samples, leading to aggregation [4]. The average diameter size of cellulose nanofibres particles were $168.9 \mathrm{~nm}$ (acid hydrolysis), $85.4 \mathrm{~nm}$ (Ultra-Turrax and microfluidizer) and $64.6 \mathrm{~nm}$ (TEMPO-oxidation and microfluidizer). 
Banana nanofibres treated with a combination of chemical and mechanical treatment shows the lowest average particle size diameter: $64.6 \mathrm{~nm}$. The fibrils obtained from the combination of TEMPOoxidation and high pressure microfluidisation were much finer compared to the fibrils treated solely with chemical or mechanical treatments. TEMPO-oxidation helps in separating the packed fibrils facilitating subsequent breakdown due to the high shear experienced from microfluidisation. Furthermore, the fibres swollen due to mercerisation treatment should cause higher friction and shear forces in the capillary during microfluidisation, resulting in finer and shorter nanofibrils [5].

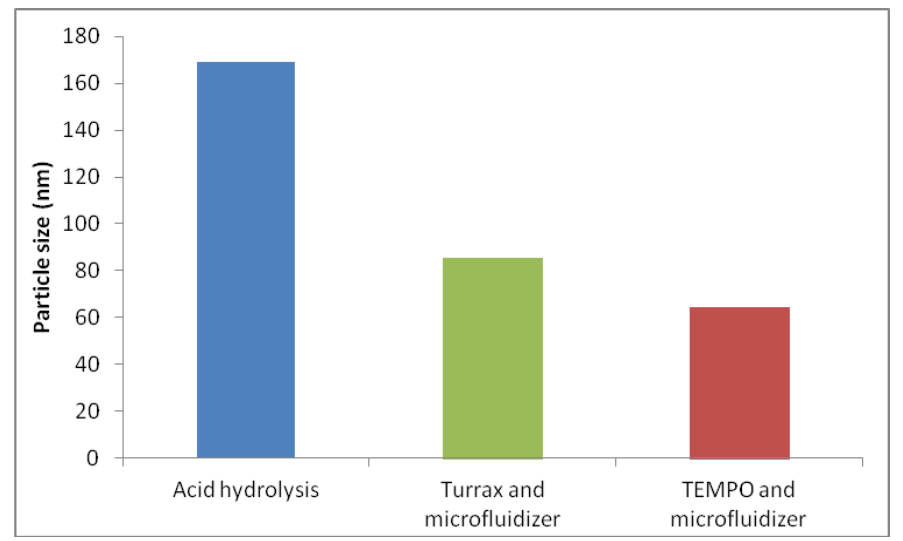

Figure 1. The diameter size distribution of banana nanofibres particles treated with various treatments.

\subsection{Zeta potential analysis}

Figure 2 shows the negatively charged zeta potential of cellulose nanofibres derived from banana fibre. Cellulose nanofibres prepared with combination of TEMPO-oxidisation and microfluidizer treatment gives the greatest negative zeta potential values: $-27 \mathrm{mV}$ showing that these nanofibres were in a more stable dispersed state compared to other samples. This is probably a consequence of the smaller average diameter size particles exhibited from this sample which results in a decrease in particle mass, leading to higher velocity movement and corresponding larger zeta potential.

A recent report by Satyamurthy et al. showed a high zeta potential of $-69.7 \mathrm{mV}$ on the surface of cellulose nanowhiskers prepared by sulphuric acid hydrolysis indicating the presence of sulphate groups in the crystallite [6]. This results in stability of the colloidal suspension of cellulose [7-8]. However in the present investigation, the zeta-potential values exhibited from the sulphuric and hydrochloric acid hydrolysed samples were less negative compared to the previously mentioned study. This suggests a lower level of sulphate groups on cellulose nanofibres, a conclusion supported by lack of bound sulphate groups seen in the FTIR spectra at $1210 \mathrm{~cm}^{-1}$ that will be presented later.

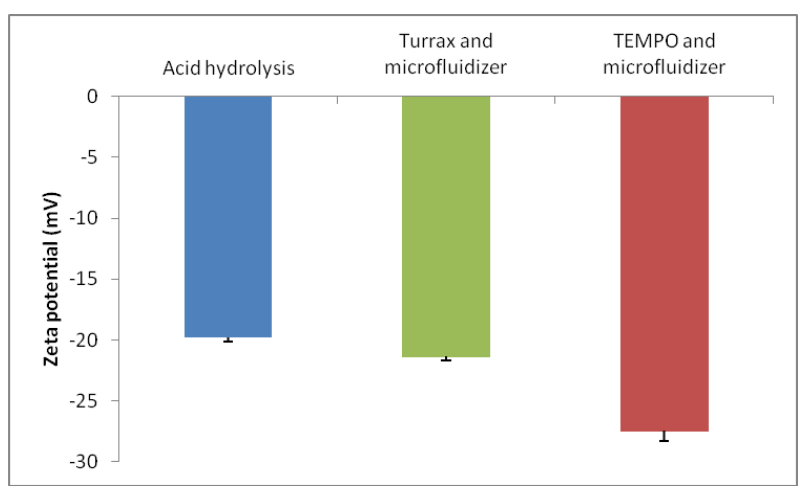

Figure 2. The zeta potential values of cellulose nanofibres derived from banana fibre treated with various treatments. 


\subsection{FTIR analysis}

Figure 3 shows the diffuse reflection FTIR spectra and results for untreated banana fibre and banana nanofibres over 4000 to $650 \mathrm{~cm}^{-1}$ wavenumbers. With the nanofibres, there is a significant decrease in peak intensity at 3340 and $2900 \mathrm{~cm}^{-1}$, corresponding to vibration of $\mathrm{OH}$...O hydrogen bonding and antisymmetric and symmetric vibration of $\mathrm{CH}_{2}$ groups respectively [9]. The presence of peaks at 1445, 1370,1160 to 1030 and $660 \mathrm{~cm}^{-1}$ corresponds to the in-plane deformation of $\mathrm{CH}_{2}$, in-plane deformation of $\mathrm{OH}, \mathrm{C}-\mathrm{O}$ stretching from ether groups, rocking vibration of $\mathrm{CH}_{2}$ and out of plane deformation of $\mathrm{OH} . . \mathrm{O}$ hydrogen bonding respectively [10-11]. The presence of hemicellulose and lignin are also evident in the region of 1060 to $1732 \mathrm{~cm}^{-1}$ respectively for banana fibre. The disappearance of the small sharp band at $1732 \mathrm{~cm}^{-1}$ and general decrease in peak intensities in the region 1060 to $1733 \mathrm{~cm}^{-1}$ was observed in banana nanofibres. This indicates that most of the hemicellulose was removed during the chemical and mechanical treatments. The peaks at 1733 and $1242 \mathrm{~cm}^{-1}$ in untreated fibre can be attributed to acetyl and uronic ester $(\mathrm{C}=\mathrm{O})$ groups from pectin, hemicelluloses along with the ester linkage of carboxylic group of ferulic and $\rho$-coumaric acids of lignin $[9,11,12]$.

However the acid hydrolysis treated nanofibre showed no absorbance in this band and a general decrease in peak intensities in the region of 1600 to $1750 \mathrm{~cm}^{-1}$. This indicates that most of the hemicelluloses and lignin have been extracted during hydrolysis process [4]. The introduction of sulphate groups is presented at $1210 \mathrm{~cm}^{-1}$, however the acid hydrolysis treated nanofibres in this study did not show absorptions corresponding to sulphate groups at $1210 \mathrm{~cm}^{-1}$ resulting from neutralisation by $\mathrm{NaOH}[4]$.

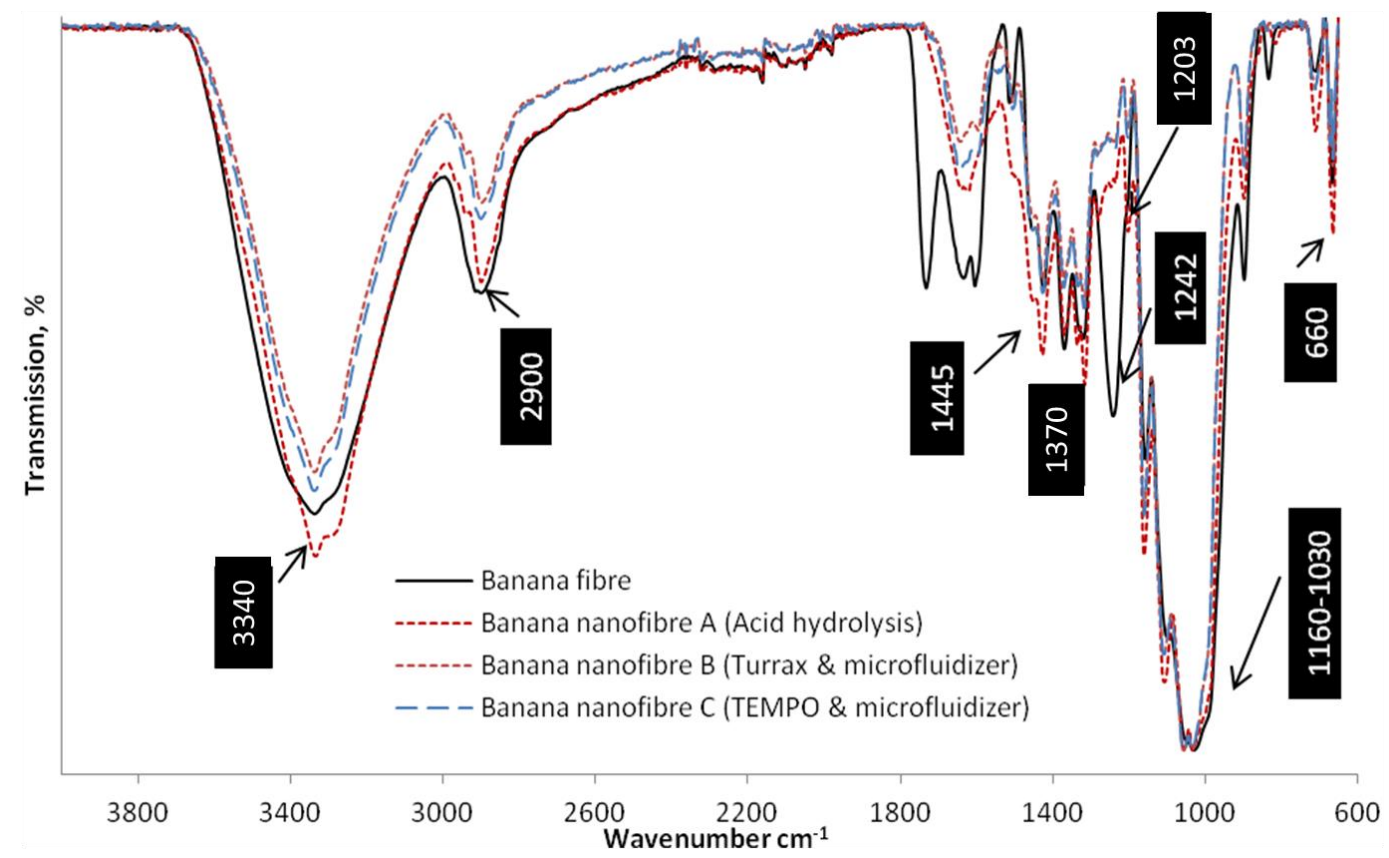

Figure 3. IR spectra of banana fibre and banana nanofibre made by using acid hydrolysis, Turrax and microfluidizer and TEMPO and microfluidizer.

\section{Conclusions}

The objective of this investigation was to determine an optimum methodology for biorefining of cellulose nanofibres. To this end, cellulose nanofibres were successfully derived from banana fibre using three different strategies; chemical treatment (acid hydrolysis), mechanical shearing (high 
dispersion Turrax mixer and high pressure microfluidizer) and combination of chemical and mechanical treatments (TEMPO-oxidation and high pressure microfluidizer). Combination of high pressure microfluidisation with TEMPO-oxidation treatment gave the most promising results, resulting in finer cellulose nanofibrils. TEMPO-oxidation was considered to facilitate the fibre breakdown process by reducing the strong intermolecular interaction within microfibril bundles.

\section{Acknowledgment}

We wish to acknowledge Research Management, Innovation \& Commercialization Centre (RMIC), UniSZA, for the financial support of this work through Special Research Grant Scheme (UniSZA/2017/SRGS/11).

\section{References}

1. C. Lew, W. Murphy and G. McNally, "Preparation and properties of polyolefin-clay nanocomposites," Polymer Engineering \& Science, vol. 44, (6), pp. 1027-1035, 2004.

2. S. Mukhopadhyay et al, "Banana Fibres - Variability and Fracture Behaviour," Journal of Engineered Fibres and Fabrics, vol. 3, (2), pp. 39, (2008).

3. A. Bhatnagar and M. Sain, "Processing of Cellulose Nanofiber-reinforced Composites," Journal of Reinforced Plastics and Composites, vol. 24, (12), pp. 1259-1268, August 01, (2005).

4. S. Sharma, "Biorefining of perennial ryegrass for the production of nanofibrillated cellulose," RSC Adv., (2012).

5. A. K. Mohanty, M. Misra and L. T. Drzal, Natural Fibers, Biopolymers, and Biocomposites. CRC, (2005).

6. P. Satyamurthy et al, "Preparation and characterization of cellulose nanowhiskers from cotton fibres by controlled microbial hydrolysis," Carbohydr. Polym., vol. 83, (1), pp. 122-129, (2011).

7. D. Bondeson, A. Mathew and K. Oksman, "Optimization of the isolation of nanocrystals from microcrystalline cellulose by acid hydrolysis," Cellulose, vol. 13, (2), pp. 171-180, (2006).

8. R. Marchessault, F. Morehead and M. J. Koch, "Some hydrodynamic properties of neutral suspensions of cellulose crystallites as related to size and shape," J. Colloid Sci., vol. 16, (4), pp. 327-344, (1961).

9. A. Alemdar and M. Sain, "Isolation and characterization of nanofibers from agricultural residuesWheat straw and soy hulls," Bioresour. Technol., vol. 99, (6), pp. 1664-1671, (2008).

10. E. Qua et al, "Preparation and characterisation of cellulose nanofibres," J. Mater. Sci., vol. 46, (18), pp. 6029-6045, (2011).

11. X. Sun et al, "Characteristics of degraded cellulose obtained from steam-exploded wheat straw," Carbohydr. Res., vol. 340, (1), pp. 97-106, (2005).

12. M. Sain and S. Panthapulakkal, "Bioprocess preparation of wheat straw fibers and their characterization," Industrial Crops and Products, vol. 23, (1), pp. 1-8, (2006). 